\title{
Desarrollo de habilidades de comprensión lectora en inglés -como lengua extranjera- en estudiantes de quinto de primaria ${ }^{1}$
}

\author{
María del Carmen Guarín Ramírez ${ }^{2}$ \\ María Isabel Ramírez Rojas ${ }^{3}$
}

Recibido: $21-07-2017$

Aceptado: 10-11-2017

\section{RESUMEN}

En el presente artículo se describe el proceso investigativo cuyo objetivo fue desarrollar habilidades de comprensión lectora en inglés -como lengua extranjera- en estudiantes de grado quinto, de una institución pública del municipio de Cartago, Valle del Cauca. Este se abordó bajo el modelo de lectura interactiva, que concibe la comprensión textual como un proceso de interacción entre el lector y el texto, se fundamentó la propuesta desde los aportes de Solé (1998); Jouini (2005); Van Dikj \& Kintsch (1983); Cummins (1983) y Anderson (1984), entre otros. El tipo de estudio fue cualitativo donde a través del enfoque de estudio de caso se analizó cómo los estudiantes desarrollaban las habilidades de lectura comprensiva en inglés. El instrumento de análisis fue una unidad didáctica

1. Trabajo clasificado como artículo de investigación. Producto de la línea de investigación Didáctica de las Ciencias Sociales de la Maestría Enseñanza de las Ciencias de la Universidad Autónoma de Manizales.

2. Candidata a Magister en Enseñanza de las Ciencias por la Autónoma de Manizales. Licenciada en Básica Primaria con énfasis en Artística de la Corporación Universitaria Minuto de Dios. Labora en la institución Educativa Sor María Juliana del municipio de Cartago, Valle del Cauca, como docente de primaria. Correo electrónico: mariacguarin1@gmail.com ORCID: 0000-0002-2053-7249

3. Magister en Enseñanza de las Ciencias Universidad Autónoma de Manizales. Magister en Orientación y Asesoría Educativa Universidad Externado de Colombia. Especialista en Desarrollo Intelectual y Educación Fundación Alberto Merani -Universidad Católica de Manizales. Licenciada en Lenguas Modernas Universidad de Caldas. Integrante del Grupo de Investigación Cognición y Educación. Docente Investigadora Departamento de Educación. Universidad Autónoma de Manizales. Antigua Estación del Ferrocarril. Manizales.

Correo electrónico: miramirez@autonoma.edu.co

ORCID: 0000-0002-7502-271X 
titulada -Healthy Food- compuesta por un instrumento de ideas previas aplicado como pre test y post test y cuatro secuencias que contenían ocho cuestionarios que sustentaron el análisis de la información.

Palabras clave: Habilidades, comprensión de lectura, comprensión de lectura en inglés, unidad didáctica.

\title{
Development of reading comprehension skills in English - as a foreign language - in fifth grade students
}

\begin{abstract}
This article describes the research process whose objective was developing reading comprehension skills in English - as a foreign language - in fifth grade students from a public school in the municipality of Cartago (V), Colombia. This proposal was based on the contributions of Solé (1998); Jouini (2005); Van Dijk \& Kintsch (1983); and Cummins (1983); among others, from the perspective of interactive reading that understands textual comprehension as a process of interaction between the reader and the text. The type of study was qualitative based on a case study approach to analyse how students developed comprehension reading skills in English. The instrument of analysis was a didactic unit entitled -Healthy food-composed by an instrument of previous ideas applied as pre-test and post-test and four sequences containing eight questionnaires that supported the analysis of the information.
\end{abstract}

Keywords: skills, reading comprehension, reading comprehension in English, didactic unit.

\section{Introducción}

El inicio del Siglo XXI se ha caracterizado por avances científicos y tecnológicos que han impactado la vida cotidiana; a lo que debe agregarse el fenómeno de la globalización y de la apertura económica. Todo ello ha provocado diversos cambios y nuevos retos para la humanidad en 
los que se incluye que el sujeto que pretende ubicarse activamente y con buen nivel de vida en el Siglo XXI, debe enfrentar gran cantidad de información escrita en inglés, debido a que la economía globalizada, la ciencia, la tecnología, la educación, el comercio y otras muchas y diversas actividades requieren este idioma.

Esta realidad hace evidente una problemática que comparten muchos países del mundo: el déficit de comprensión de lectura en inglés; problema que se manifiesta en estudiantes de todos los ciclos escolares. McNamara (2004), expresa al respecto, que la cantidad de estudiantes que presentan dificultades para responder apropiadamente a preguntas concernientes a textos escritos en inglés es significativa y más aún cuando son monolingües. Esta problemática ha despertado especial interés de profesores e investigadores universitarios quienes han desarrollado algunos estudios y elaborado propuestas metodológicas que tienden a favorecer el desarrollo de las habilidades de lectura comprensiva en este idioma. A pesar de ello, en el contexto de la educación básica y específicamente en los grados de primaria, estas investigaciones han sido escasas.

Cabe destacar que actualmente los estudiantes muestran gran interés en lo que son las artes, la literatura, el cine y la música, especialmente esta última, pues son parte de los aspectos culturales que los motivan porque tienen afinidad con sus gustos personales; de modo que ven que la cultura, en general, sin especificar una de ellas, motivan el aprendizaje del inglés a nivel global, por los beneficios que este le puede traer a una persona a nivel laboral, estatus social, poder y propósitos del lenguaje. (Beltrán, 2015, p. 355). Motivación que es relevante al momento de proponer investigaciones en esta disciplina del saber.

Al revisar las investigaciones realizadas en Colombia en este campo, no se encontraron trabajos enfocados al desarrollo de habilidades de lectura en inglés en la básica primaria, a pesar de que desde los entes gubernamentales se han dado directrices que buscan mejorar los procesos de enseñanza y aprendizaje de esta disciplina del saber desde los primeros grados escolares ${ }^{4}$. Es así como en el Programa Nacional de

\footnotetext{
4. A partir de la expedición de la Ley General de Educación de 1994 (Ministerio de Educación Nacional, 1994, Art. 21 literal m), se incluyó dentro de las áreas obligatorias la enseñanza de una lengua extranjera en el nivel escolar de primaria. En el año 2004, se formuló el Programa Nacional de Bilingüismo 2004-2019, que incluye los nuevos estándares de competencia comunicativa en inglés como lengua extranjera, Posteriormente esta propuesta de bilingüismo se reforma tomando el nombre de Programa Nacional de inglés Colombia Very Well 2015-2025 (Ministerio de Educación Nacional, 2014).
} 
inglés 2015-2025 del Ministerio de Educación Nacional (MEN), publicado en 2014, se afirma que:

Consolidar una educación de calidad implica asegurar que en las aulas los niños y jóvenes están desarrollando las competencias que necesitan para la vida y que saben cómo usar esas competencias en su cotidianidad. El manejo de una lengua extranjera es sin duda una habilidad que empodera a los individuos, les brinda mayores oportunidades de acceso al conocimiento y a otras culturas y los hace más competitivos.

(p. 3).

Para conseguir este propósito, desde el año 2006, el MEN publicó los estándares básicos de competencia en lengua extranjera: inglés, que consideran como habilidades de comprensión lectora de los estudiantes de grado cuarto y quinto de primaria las siguientes: comprender descripciones cortas ubicando los lugares y momentos en que suceden las acciones; identificar las acciones en una secuencia corta de eventos; utilizar gráficas para representar la información más relevante de un texto; identificar elementos culturales como nombres propios y lugares, en textos sencillos; leer y entender textos auténticos y sencillos que versen sobre acontecimientos concretos asociados a tradiciones culturales que conoce, así como para reconocer en un texto narrativo corto, aspectos relacionados con el qué, quién, cuándo y dónde (Ministerio de Educación Nacional, 2006).

Se aborda el concepto de comprensión lectora en inglés por considerar que el déficit de comprensión de lectura en inglés es una problemática que se manifiesta en estudiantes de todos los ciclos escolares, en términos de McNamara (2004), la cantidad de estudiantes que presenta dificultades para responder adecuadamente a preguntas referentes a textos escritos en inglés es significativa y se agudiza cada día más.

De acuerdo con Solé (1998), el proceso de comprensión lectora que cada uno realiza depende del texto que tenga delante y de otras cuestiones propias del lector, entre las que se pueden señalar el conocimiento previo, los objetivos que la presiden y la motivación que se siente hacia dicha lectura. La comprensión de lectura que hace el sujeto en una lengua extranjera, determina en primer lugar, el nivel de apropiación de conceptos correspondientes a determinada disciplina, al tiempo que se aprende la lengua extranjera, en segundo lugar, posibilita 
la enseñanza contextualizada, en tercer lugar, motiva a encontrar el sentido e importancia al aprendizaje de una segunda lengua y cuarto, se aprenden estrategias para comprender los textos de acuerdo con las estructuras propias de los mismos textos. De allí, surge la pregunta: ¿Cómo desarrollar habilidades de comprensión lectora en inglés como lengua extranjera- en estudiantes de quinto de primaria, de una institución pública del municipio de Cartago, Valle del Cauca?

Para dar respuesta a esta pregunta, en el estudio se fundamentaron las categorías conceptuales de: comprensión lectora; niveles de comprensión de lectura y comprensión lectora en inglés. Además, desde el marco de la enseñanza de las ciencias en perspectiva sociocientífica, la ciencia en el aula y los problemas socialmente relevantes, se partió de un problema que en los últimos años ha afectado a un número importante de personas en todo el mundo y específicamente a los niños, la obesidad infantil. A partir de este, se diseñó e implementó una unidad didáctica titulada -Healthy Food- la cual se estructuró bajo el modelo de enseñanza del inglés a través de contenidos disciplinares, desarrollada por Brinton, Nieve y Wesche (1989).

\section{Comprensión lectora}

La comprensión de lectura es una actividad de orden superior que ha posibilitado a los sujetos apropiarse de los conocimientos construidos a través de la historia de la humanidad y a los que hoy se tiene acceso desde las diferentes disciplinas científicas $y$, a través de los medios de comunicación, especialmente en internet. Esta práctica es fundamental para contribuir en el desarrollo del pensamiento crítico de los educandos, por lo que ha sido objeto de numerosos estudios desde diferentes campos disciplinares entre los que se encuentran: la psicología cognitiva, las ciencias de la educación y las que forman parte de las ciencias cognitivas (lingüística, filosofía, psicología del aprendizaje, inteligencia artificial y antropología, entre otras).

En este sentido, desde las ciencias cognitivas se argumenta que comprender implica construir modelos o representaciones coherentes entre lo que se escucha, o se lee con las representaciones presentes en los sujetos, para lo cual dichos sujetos realizan inferencias para conseguirlo (Sánchez, 2009). Al respecto De Vega (2005), expresa que cuando un sujeto se enfrenta a un texto escrito, a nivel cerebral se 
congregan sistemas de: memoria, atención, procesos de codificación y de percepción y operaciones inferenciales. Esta teoría la comparten también autores como Solé (1987), Cassany (2005) y Jouini (2005).

Otros estudios, se han orientado hacia lo representacional en la mente del lector. Los procesos y estrategias cognitivas y metacognitivas que le permiten la autorregulación del aprendizaje (Graesser, Gernsbacher y Goldman, 2000; Graesser, Millis y Zwaan, 1997; Parodi, 2002; Van Dijk y Kintsch, 1983; citados por Hernández, 2005).

Comprender es entonces, dar significado a un texto a través de un proceso de interacción entre el lector y el texto; interacción que, desde luego, está dominada por el lector, donde, por ejemplo, la interacción que va del texto al lector es creada y dirigida por el lector. Kintsch y Van Dijk (1983), expresan que en la lectura median aspectos tanto del entorno educativo, social y cultural del lector y del escritor, como de las estructuras mentales del lector.

En este orden de ideas, los estudios sobre comprensión de lectura, han buscado ayudar a los estudiantes a mejorar sus desempeños como lectores; para lo cual, se han implementado varios modelos a lo largo de la historia. Estos modelos han sido básicamente organizados en tres categorías: (a) Primarios, superficiales o ascendentes (botton-up), que conceden importancia, principalmente, al nivel de percepción; (b) Secundarios, profundos o descendentes (top-Down), que inician el proceso en niveles superiores, donde los conocimientos previos del lector son fundamentales para comprender los textos; (c) Interactivos, que no solamente integran los dos modelos anteriores, sino que consideran otros parámetros como lector, texto y contextos, tanto del lector como del escritor, y de una manera tácita a quien enseña. (Alonso y Mateus, 1985).

Desde la posición teórica del modelo interactivo, leer es el proceso mediante el cual se comprenden las ideas de un texto porque, entre otras cosas, se comprende el lenguaje escrito. Por lo tanto, como lo expresa Solé (1998), la lectura es una actividad cognitiva compleja; el lector un procesador diligente quien, a partir de sus conocimientos previos, esquemas mentales, planteamiento de objetivos claros de lectura y el uso de estrategias de comprensión, construye el significado del texto.

En este proceso, el lector pasa por distintos niveles, -literal, inferencial y crítico-. Según lo expresado por Van Dikj y Kintsch (1983), en el nivel 
literal el lector reconoce el código de superficie, es decir, interpreta el léxico y lo analiza a nivel sintáctico. En nivel inferencial, reconoce el texto base, lo que le exige conectar las proposiciones e interpretarlas a nivel local (micro estructura) y a nivel global (macro estructura), para lo cual utiliza estrategias de supresión, generalización y construcción y, en el nivel crítico hace una abstracción del texto para proponer un nuevo escrito donde expone sus propios argumentos.

Jouini (2005), al respecto plantea que:

En el proceso de comprensión de textos escritos se pueden tres grandes niveles que son dependientes y que interactúan entre ellos: a) El lector de un texto debe reconocer las palabras a través de la codificación de cada una de ellas y el acceso a su diccionario interno que le proporciona su significado; b) El lector debe contar con un analizador sintáctico que relaciona las palabras de cada oración y las proposiciones entre sí; c) En el nivel de análisis semántico, el lector debe ser capaz de ligar unas proposiciones con otras para formar una representación coherente de lo que se está leyendo como un todo, inferir las relaciones semánticas entre las diferentes unidades textuales y poder jerarquizar e interrelacionar las ideas del texto a nivel global. (p.101).

Como se puede observar en las dos teorías anteriores, en el tránsito por los tres niveles de lectura el lector parte del reconocimiento de las palabras, las vincula a nivel proposicional y luego las interpreta con la totalidad del escrito, para lo cual integra: sus conocimientos, capacidad cognitiva, estado emocional y motivacional. Estas acciones llevan a concluir que cada texto tiene múltiples interpretaciones dependiendo de quien lo lee.

\section{Comprensión lectora en inglés}

Según lo expresado por McNamara (2004), la actividad lectora es compleja, y se vuelve aún más compleja cuando se trata de una lengua que no es la lengua materna, ya que en el proceso lector se concatenan aspectos psicolingüísticos que requieren, no solamente de conocimientos gramaticales, sino de actitud abierta y dinámica por 
parte del lector que lo lleven, a través de la lectura a entablar un diálogo profundo con el texto hasta proponer un nuevo escrito bajo sus propios argumentos.

En el transcurso de la historia se han propuesto variados métodos para mejorar los procesos lectores y la adquisición de un segundo idioma en los estudiantes de los diferentes niveles educativos; ejemplo de estos son: a) El método audiolingüe (MAL), basado en el enfoque conductista en el cuál predomina la memorización de vocabulario y repetición de diálogos (Jaimes, 2003); b) el método natural de Krashen \& Terrel (1983), en el que se asegura que si el sujeto está inmerso en el idioma y establece una relación dialógica constante con este, se produce el aprendizaje y el dominio del mismo. En cuanto a la lectura, según Hernández (1999), este método proponía leer textos que sirvieran para el uso de la lengua en la cotidianidad por ejemplo leer un menú o una rutina de actividades; c) el comunicativo, a través del cual se pretende que los estudiantes conozcan aspectos fundamentales de la estructura de la lengua para que puedan hacer un uso correcto de la misma. Al igual que en la propuesta de Krashen \& Terrel (1983), al estudiante se le pone en situaciones reales de uso del idioma y, además, se le entregan fundamentaciones teóricas de tipo gramatical. (Muñoz, 2009).

A partir del enfoque comunicativo han surgido otras propuestas como la teoría de la interdependencia lingüística propuesta porCummins (1981), quien expresa que si un sujeto aprendiz tiene una exposición a su primera lengua con estímulos suficientes para que esta se desarrollara de manera adecuada, entonces este aprendiz, al estar en un contexto académico para aprender un segundo idioma lo haría recurriendo a los esquemas adquiridos en el aprendizaje de su primera lengua; de ahí la importancia de realizar excelentes procesos de lectura en la lengua nativa.

Esta propuesta tiene relación directa con la teoría de los esquemas de Piaget (1967), la cual dió origen a los postulados del aprendizaje significativo de Ausubel y la teoría socio-cognitiva de Vygotsky. Anderson (1984), citado por Salazar (2006), incorpora esta al escenario lingüístico, expresando que todos los conocimientos adquiridos por el ser humano, desde que nace, se encuentran reunidos en estructuras mentales denominadas esquemas, los cuales se van enriqueciendo con los aprendizajes que se adquieren a través de los años.

En cuanto a la teoría de los esquemas con relación a comprensión 
lectora en una segunda lengua, Fernández \& Montero (2005), arguyen que, dado que el texto no tiene significado en sí mismo, sino que este tiene un significado para quien escribe y otro para quien interpreta, se hace necesario que el lector cuente con esquemas de comprensión que le posibiliten una interpretación asertiva; de ahí la importancia de dotar de habilidades o estrategias lectoras a los aprendices, porque si es hábil para interpretar textos en su primera lengua, podrá recurrir a estas estrategias y trasponerlas para que la interpretación textual en un segundo idioma le sea más cercana.

Al respecto Jouini (2005), expresa que para ayudar a un aprendiz de una segunda lengua a ampliar sus ideas alternativas se le debe exponer constantemente a relacionarse con dicha lengua y que una de las mejores formas es proponerle, desde el principio de su contacto con la nueva lengua, lecturas de interés que enriquezcan su conocimiento sobre las diferentes estructuras sociales y culturales de la lengua meta.

En relación con las teorías, a finales del siglo $X X$, surge la propuesta por Brinton, Nieve y Wesche (1989), llamada Instrucción Basada en Contenidos (CBI - Content Based Instruction), que fue implementada en dos contextos académicos distintos dentro de los EEUU. Estos autores, la definen como "la integración de un contenido específico, por ejemplo de matemáticas, ciencias o estudios sociales. Se refiere a la enseñanza simultanea del saber disciplinar con el desarrollo de habilidades en la segunda lengua" (Brinton, Nieve y Wesche, 1989, p.2 citados por Shang, 2016). En este enfoque, el profesor de inglés conecta temas de otras asignaturas con el fin de ayudar a los estudiantes a desarrollar habilidades de comprensión lectora en inglés, desde el contenido de los textos disciplinares. Lo importante en este enfoque es comprender el texto en sí mismo y no sus estructuras gramaticales (Cassany, 1990).

\section{Metodología}

La investigación es un estudio cualitativo que según Hernández, Fernández y Baptista (1991), para el caso de los estudios en el campo educativo se presenta como una alternativa a seguir para comprender los problemas de enseñanza y aprendizaje. El enfoque fue el estudio de caso;

5. Cummins (2005), citado por Freeman \& Freeman (2008), expresa, además, que el lenguaje académico es cognitivamente demandante; por lo que el maestro debe estar en capacidad de ofrecer a los estudiantes los estímulos y las ayudas necesarias para que puedan desarrollar las habilidades tanto de escritura como de comprensión cuando se enfrentan a los diferentes tipos de textos. 
como lo expresa Yin (1981), es un método adecuado cuando se trata de responder preguntas de ¿cómo? y ¿por qué? y se utiliza cuando se quiere conocer una situación compleja. Para efectos de esta investigación se consideraron tres casos con el fin de conocer a profundidad cómo estos estudiantes desarrollaban habilidades para comprender textos en inglés.

El estudio tuvo en cuenta los siguientes momentos:

Primer momento de selección de la temática a trabajar, la realización de un cuestionario, para explorar las ideas previas. Posterior a ello, se diseñó la unidad didáctica, que a su vez contenía cuatro secuencias. Una vez finalizada la unidad didáctica, se aplicó el cuestionario inicial como postest. Dicha unidad didáctica se aplicó a los 42 estudiantes de grado quinto de la institución educativa Sor María Juliana de la ciudad de Cartago, Valle del Cauca.

El segundo momento, corresponde al análsis propiamente dicho, en donde se hizo un análisis de los hallazgos en el grupo de estudiantes con el cual se trabajó, para luego profundizar en el análisis de los tres casos escogidos.

Para la recolección de la información, se emplearon el cuestionario de ideas previas utilizado como pretest, además en cada una de las secuencias didácticas se aplicaron dos pruebas de lectura, una al iniciar y la otra al finalizar cada una de las secuencias de la unidad didáctica y por último, se aplicó el cuestionario inicial como postest. Los cuestionarios iniciales y finales, pretendían hacer seguimiento a los niveles de lectura en lengua extranjera que poseían los estudiantes.

\section{Resultados}

De acuerdo con los momentos del proceso investigativo, se realizó un análisis descriptivo de la información recolectada, para ello se sistematizó la información de cada uno de los instrumentos aplicados en matrices de análisis.

A partir de esta sistematización, se establecieron criterios para valorar los niveles de lectura literal e inferencial y se diseñaron protocolos de evaluación por cada cuestionario y por cada pregunta planteada. 
A continuación, se exponen los resultados más destacados.

\section{Instrumento de ideas previas aplicado como pre-test y post-test}

Afirmación: "My good health depends on the right balance between what I eat and how much I move".

E1: I Agree "Sí estoy de acuerdo, porque mi buena nutrición depende del balance en el movimiento"

E2: I don't understand. "No entiendo, porque hay palabras que se me olvidaron y por eso no sé lo que dice".

E3. I don't understand. "No entiendo porque no me dieron clase de inglés el año pasado y no sé lo que dice".

\begin{tabular}{|l|}
\hline Afirmación: "Eating saturated fats is not good for my heart". \\
\hline E1: I don't understand. "Marqué la D porque no entiendo bien lo que dice". \\
E2: I disagree "Estoy en desacuerdo porque la grasa saturada es mala para la salud". \\
E3. I don't understand. "No entiendo porque no me dieron clase de inglés el año pasado".
\end{tabular}

Tabla 1. Respuestas al instrumento de ideas previas.

El instrumento de ideas previas se diseñó bajo el modelo de escala de Likert donde se proponían diez afirmaciones escritas en inglés con cuatro opciones de respuesta: "I Agree___. I disagree___. I don't know I don't understand ." Una vez seleccionada la respuesta, los estudiantes debían justificar dicha elección. (utilizando su lengua materna).

En la tabla 1 se presentan algunas de las respuestas aportadas por los estudiantes.

Como se puede observar, en las justificaciones que presentaron los estudiantes durante el pre-test se evidencia que no lograron decodificar los textos. Esto significa según la propuesta de Van Dijk y Kintsch (1983), que no accedieron al reconocimiento del código de superficie donde se le exige al lector el reconocimiento del léxico y la interpretación sintáctica del mismo. Para Jouini (2005), los problemas que se presentan en la comprensión de lectura se deben a varios factores: a). "Dificultades para comprender una palabra"; b). "Dificultades para comprender una oración"; c). "Dificultades para comprender como una oración se relaciona con otra" y d). "Dificultades para comprender como encaja el texto completo". Lo expresado por el autor se ve reflejado en las respuestas de los estudiantes, quienes en la mayoría de las afirmaciones respondieron no entender debido a la falta de comprensión del vocabulario y cuando 
expresaban estar de acuerdo con la afirmación, en las justificaciones se pudo constatar claramente que traducían algunas palabras, pero no las contextualizaban con el sentido global de los textos.

Del análisis del instrumento de ideas previas se pudo constatar que los estudiantes presentaban dificultades severas en la comprensión lectora en inglés antes de iniciar la intervención didáctica, confirmando la necesidad de aprendizaje de este grupo de educandos, quienes, ante textos sencillos, que trataban temáticas tratadas a lo largo de la educación primaria, no accedían a la comprensión a nivel literal.

\section{Análisis y discusión de la categoría literal durante la intervención didáctica}

Durante cada secuencia se plantearon preguntas de orden literal. Al finalizar la secuencia 4, los estudiantes leyeron el texto "Good Health", del cual se plantearon siete preguntas de opción múltiple, entre las que se encontraba la que se presenta a continuación y cuya respuesta estaba explicita en el escrito: "Beans, grains, fruit and vegetables are examples of what?". Los niños debían escoger una opción y justificar la respuesta.

En la tabla 2, se presentan las respuestas.

\begin{tabular}{|c|c|c|}
\hline Texto & Opción elegida & justificaciones \\
\hline \multirow{3}{*}{$\begin{array}{l}\text { "Beans, grains, fruit and } \\
\text { vegetables are examples of } \\
\text { what?": } \\
\text { a. Unhealthy food } \\
\text { b. Foods you should eat } \\
\text { several times a day. } \\
\text { c. Foods that can cause } \\
\text { obesity. } \\
\text { d. Foods with fiber. }\end{array}$} & E1. “D” & $\begin{array}{l}\text { "Porque en el párrafo tres } \\
\text { se habla sobre una dieta } \\
\text { saludable y comida con fibra". }\end{array}$ \\
\hline & E2." D" & $\begin{array}{l}\text { "El texto y otros textos nos } \\
\text { dicen que estos alimentos } \\
\text { tienen fibra que es importante } \\
\text { para el funcionamiento del } \\
\text { intestino". }\end{array}$ \\
\hline & E3. "B" & $\begin{array}{l}\text { "Porque en el párrafo tres } \\
\text { dice que comer vegetales y } \\
\text { alimentos saludables todos } \\
\text { los días nos dará salud" }\end{array}$ \\
\hline
\end{tabular}

Tabla 2. Respuestas a pregunta de orden literal.

Al analizar las anteriores respuestas se infiere que los casos 1 y 2 , encontraron la información literal contenida en el texto. El primer caso especifica en su argumento el lugar de la lectura en el que se hablaba de los alimentos con fibra y el caso 2 , resaltó que en el texto se encontraba 
la respuesta, pero además, aportó información proveniente de sus presaberes para ampliar su argumento.

Se puede afirmar que los estudiantes E1 y E2, durante la intervención adquirieron elementos gramaticales y vocabulario que les permitió decodificar el texto y, como lo expresa Jouini (2005), decodificar no es comprender, pero si es el primer paso para interpretar un escrito. En este caso, el escrito contenía cuatro párrafos cortos y la respuesta a la pregunta solicitada estaba, como lo expresó el E1 en el tercer párrafo, lo que significó un ejercicio cognitivo de rigor para los estudiantes, dado que se trataba de un escrito en inglés.

En la respuesta del E.3, se nota que comprendió parte de la pregunta, también que tenía claridad de lo que decía la opción que eligió; sin embargo, eligió la incorrecta. Pese a este error, el estudiante también tuvo un progreso significativo en su proceso de lectura, ya que se sentía temeroso de responder las preguntas en este idioma y finalmente logró hacerlo.

\section{Análisis y discusión de la categoría inferencial durante la intervención didáctica}

A continuación, en la tabla 3 se presentan algunas de las respuestas a las preguntas que buscaban que los estudiantes realizaran lectura inferencial.

\begin{tabular}{|c|c|c|}
\hline Texto & Pregunta & Respuesta \\
\hline $\begin{array}{l}\text { Texto 1: } \\
\text { Weight } \\
\text { Problems and } \\
\text { Obesity in } \\
\text { Children }\end{array}$ & $\begin{array}{l}\text { Explain the following sentence: } \\
\text { "Overweight children are frequently } \\
\text { teased and excluded from team } \\
\text { activities?" }\end{array}$ & $\begin{array}{l}\text { E1. "No la puedo explicar porque está } \\
\text { muy difícil" } \\
\text { E2. "A los niños con sobre peso los } \\
\text { excluyen de actividades en equipo" } \\
\text { E3. No responde }\end{array}$ \\
\hline $\begin{array}{l}\text { Texto 2: } \\
\text { Healthy Eating } \\
\text { Plate }\end{array}$ & $\begin{array}{l}\text { "What could be said about drinking } \\
\text { coke and soda?" } \\
\text { a. drink it after lunch, but not after } \\
\text { dinner. } \\
\text { b. avoids them } \\
\text { c. better to drink soda with fruits } \\
\text { artificial flavor. } \\
\text { d. drink them but with low sugar. } \\
\text { Why? }\end{array}$ & $\begin{array}{l}\text { E1. B. "porque en la imagen se muestra } \\
\text { que se debe tomar es agua". } \\
\text { E2. B. "Porque en la imagen de un plato } \\
\text { saludable, hay un vaso de agua que es lo } \\
\text { que se debe tomar, porque la cocacola es } \\
\text { una bebida negra muy mala y también las } \\
\text { otras gaseosas. } \\
\text { E3 C porque se deben tomar jugos de } \\
\text { frutas saludables. }\end{array}$ \\
\hline
\end{tabular}

Tabla 3. Respuestas a preguntas a nivel inferencial. 
Al analizar la pregunta del texto 1, se pudo observar que a pesar de haber trabajado durante el desarrollo de la primera secuencia didáctica con todo el vocabulario contenido en la oración, a los estudiantes se les dificultó interpretarlo, ya que sólo el E2, logró realizar una traducción del escrito. De las respuestas aportadas, se pudo inferir que a esta edad realizar procesos de lectura a nivel inferencial es una tarea que representa serias dificultades para los aprendices. Salvador, Gallego \& Mierés (2007), expresan que acceder a la interpretación textual exige desarrollar algunos procesos cognitivos como dar el significado apropiado a las palabras y construir primero, un significado a nivel local y luego a nivel global, lo que supone trabajo y dedicación en los aprendices. En cuanto a los educadores, como afirma Jouini (2005), les exige "plantear preguntas cuya respuesta no esté explicitada en el texto, de manera que a los estudiantes les suponga una exigencia cognitiva, que finalmente les será de gran utilidad en futuros aprendizajes" (p.102).

En el segundo texto, en el que se debía responder una pregunta de opción múltiple y justificar la respuesta, se observa que los estudiantes E1 y E2 marcaron la opción correcta y la justificación corresponde con la selección que hicieron. Los dos estudiantes pudieron inferir por qué el texto decía que la coca cola y las gaseosas en general se deben evitar, ya que hicieron alusión a que, "en la imagen del plato saludable, había un vaso de agua". En el caso del E.3, se observa que comprendió la pregunta, pero no las opciones de respuesta, lo que le impidió seleccionar la correcta. En este caso, el logro de los estudiantes se debió en gran medida a que el texto estaba acompañado de imágenes, de ahí la importancia del uso de múltiples lenguajes en la enseñanza de la lectura comprensiva en lengua extranjera.

En el tercer texto, donde se presentó la lectura -Good Health-, que contenía cuatro párrafos, se propuso que explicaran en sus propias palabras el contenido de cada párrafo.

\section{El E.1 respondió:}

"El primer párrafo habla sobre el significado de la salud para la mayoría de personas".

"El segundo párrafo habla sobre lo importante del ejercicio para estar saludables".

"El tercer párrafo habla sobre la importancia de la dieta saludable para la buena salud". 
"El cuarto párrafo habla sobre el estrés y como superarlo para mantener el cuerpo saludable".

En estas respuestas, se puede observar que el estudiante suprimió la información secundaria contenida en cada párrafo, lo que le posibilitó encontrar la idea central de cada escrito. Estas acciones corresponden con el nivel de lectura inferencial. Van Dijk y Kintsch (1983), expresan que en este nivel se reconoce el texto base; el lector logra establecer las relaciones del escrito a nivel local (que corresponde a la microestructura) y luego las relaciona para establecer la idea global del escrito, (que corresponde a la macroestructura). Se infiere entonces, que el estudiante reconoció el texto base.

El E.2 respondió la misma pregunta de la siguiente forma:
"Primer párrafo; Más personas están de acuerdo con estar saludable, aunque muchas opiniones son diferentes, hay que tomar medidas para no estar enfermo".
"Segundo párrafo: Una de las mejores formas de cuidar tu cuerpo es haciendo ejercicio, pero muchas personas no están de acuerdo, dicen que no es necesario hacer ejercicio todos los días. Solo un simple trabajo como limpiar la casa es como si estuvieras haciendo ejercicio. Otras personas hacen ejercicio todos los días como correr o nadar".
"Tercer párrafo: tener una dieta te ayuda a comer saludable. Comidas como vegetales y frutas tienes que incluirlos en la dieta. Es importante comer alimentos con fibra como: frijoles, granos, frutas y vegetales. La fibra ayuda a tu cuerpo a hacer digestión de la comida que comiste. Estando obeso estas arriesgando a tu salud".
"Cuarto párrafo, El nivel de estrés en la vida es alto. Ejercicio y deporte reduce el estrés. Otras actividades como el tai-chi, yoga y caminar también reduce el estrés".

Al analizar las respuestas proporcionadas por el E.2, se pudo evidenciar que reconoció el léxico y lo relacionó adecuadamente en el contexto de las proposiciones, esto le posibilitó responder recurriendo a la traducción parcial de cada uno de los párrafos. Esto significa que el estudiante en esta pregunta hizo lectura literal. Sin embargo, se resalta que logró interiorizar un buen número de vocabulario, lo que posibilita que siga avanzando en su proceso de lectura comprensiva. 
En cuanto al E. 3 sus respuestas fueron las siguientes:

" El primer párrafo habla de que las personas decidieron tener salud"

"En este segundo párrafo se habla del ejercicio".

"Este tercer párrafo dice de los alimentos saludables"

"En este cuarto párrafo se habla de hacer ejercicio también.

En las respuestas del E.3, se observa que detectó algunas palabras claves de los párrafos, sin embargo, cuando dice que en el primer párrafo se habla "que las personas decidieron tener salud", se confundió en la interpretación, porque esta no era la idea expresada en el texto. Esto mismo ocurrió con la respuesta que dio sobre el párrafo cuatro, donde no pudo establecer las relaciones de la lectura con sus presaberes, quizás porque confundió la información. En cuanto a los párrafos dos y tres, es correcto que trató el tema del ejercicio y de la alimentación saludable, pero, al no aportar ninguna otra idea, se infiere que el estudiante con algunas palabras que comprendió dio cumplimiento al ejercicio solicitado, pero no logró realizar una interpretación de los párrafos.

\section{Conclusiones}

El objetivo general propuesto en la presente investigación propendía por desarrollar habilidades de comprensión lectora en inglés -como lengua extranjera- en estudiantes de quinto de primaria. Cumplido este objetivo, se concluye que el proceso de lectura comprensiva en una lengua extranjera, no es una tarea fácil y requiere de tiempo, planeación y conocimiento por parte del educador. En este sentido, se pudo comprobar, desde la práctica de enseñanza del inglés en los primeros grados escolares, que frecuentemente la enseñanza de este idioma se limita a la memorización de vocabulario que, aunque es necesario, no genera aprendizaje, porque los estudiantes no logran darle significado apropiado en el contexto de global del escrito.

Sin embargo, con este estudio se hizo evidente como a través del diseño, aplicación y evaluación de unidades didácticas se potencia el desarrollo de habilidades de lectura comprensiva en inglés -como lengua extranjera- ya que su estructura parte del conocimiento de cómo inician el proceso los educandos con la aplicación del pre-test; luego durante 
la intervención, se monitorea dicho proceso a través de la evaluación constante tanto de los aprendizajes como de la práctica de enseñanza y finalmente se evalúa como se transformó el conocimiento a través del post-test. Además, estructurar la unidad didáctica a partir de una temática disciplinar, es relevante, pues posibilita que los estudiantes se enfrenten a textos auténticos que son significativos para ellos y a la vez les exige realizar acciones de tipo cognitivo que ayudan en la adquisición de aprendizajes profundos.

Es evidente, además, que para los estudiantes es más fácil responder a preguntas de orden literal que inferencial, ya que este es un proceso complejo, más aún al tratarse de una lengua extranjera, razón por la cual, desde los primeros acercamientos a la lectura los docentes deben proponer preguntas de tipo inferencial, de manera que los educandos puedan ir desarrollando la capacidad de leer comprensivamente, pues la garantía de comprensión, es la capacidad del lector para hacer inferencias. (Jouini, 2005).

Finalmente, este trabajo se convierte en fundamento de futuras investigaciones, ya que en primer lugar, ha sido un campo poco explorado en los niveles de básica primaria, y, además se evidencia que el trabajo de la lectura comprensiva en lengua extranjera, desde los primeros grados escolares, posibilita que los niños amplíen el vocabulario, lo interpreten en contextos escritos y le encuentren sentido y utilidad al idioma; factores indispensables para garantizar el aprendizaje. 


\section{Referencias bibliográficas}

Acevedo Zapata, S. (2015). La formación de profesionales desde la reflexión en la acción con elementos fundamentales de la pedagogía crítica. Revista De Investigaciones UNAD, 14(1), 151 - 169. doi:http://dx.doi.org/10.22490/25391887.1350

Alonso, J. \& Mateus S., M. (1985). Comprensión lectora: modelos, entrenamiento y evaluación. Infancia y aprendizaje: Journal for the study of education and development, 31-32, 5-30. Recuperado de https://dialnet.unirioja.es/servlet/ articulo?codigo $=667401$.

Beltrán (2015). Influencia cultural en la motivación para el aprendizaje del inglés como lengua extranjera Revista Interamericana de Investigación, Educación y Pedagogía, Vol. 8, Num. 2, 341 - 371. Recuperado de revistas.usta.edu.co/index.php/riiep/article/ download/2879/2751

Cassany, D. (1990). Enfoques didácticos para la enseñanza de la expresión escrita. Comunicación, lenguaje y educación 6, 63-80. Recuperado de https://www.upf.edu/ pdi/dtt/daniel_cassany/enfoqes.htm.

Cummins, J. (1981). The role of primary language development in promoting Educational success for language minority students, in California State Department of Education (ed.). Schooling and language minority students. Theoretical Framework. Los Angeles: California State Department of Education. Recuperado de http://files.eric. ed.gov/fulltext/ED249773.pdf.

De Vega, M. (2005). Lenguaje, corporeidad y cerebro: Una revisión crítica. Signos, 38, (58). Recuperado de http://dx.doi.org/10.4067/S0718-09342005000200002.

Fernández Agüero, M. y Montero Méndez, S. (2005). La lectura en segundas lenguas: análisis de la contextualización previa y sus implicaciones metodológicas. Porta linguarum, 3,35-44. Recuperado de http://www.ugr.es/ portalin/articulos/PL_numero3/ fernandez-lectura.pdf.

Freeman, D. \& Freeman, Y. (2008). Enseñanza de las lenguas a través del contenido académico. Revista educación y pedagogía, 20(51), 97-110. Recuperado de http:// aprendeenlinea.udea.edu.co/revistas/index.php/revistaeyp/article/view/9899.

Hernández Rojas, G. (2005). La comprensión y la composición del discurso escrito desde el paradigma histórico-cultural. Perfiles Educativos, 27(107), 85-117. Recuperado de http://www.scielo.org.mx/pdf/peredu/v27n107/n107a05.pdf

Hernández S., R.; Fernández, C. y Baptista, P. (1991). Metodología de la investigación. México: McGraw-Hill. Recuperado de https://competenciashg.files.wordpress. com/2012/10/sampieri-et-al-metodologia-de-la-investigacion-4ta-edicionsampieri-2006_ocr.pdf.

Herrera, L.M. y Vásquez, A.F. (2016). Investigación escolar, formación, experiencia e interés de docentes de básica primaria. Revista Interamericana de investigación, Educación, Pedagogía. Vol. 9. Núm. 1, 125-138. Recuperado de http://revistas.usta.edu. co/index.php/riiep/article/view/3606/3501 
Jaimes Muñoz, S. (2003). El uso de textos literarios en la enseñanza del inglés en la educación secundaria. España: Universidad de Granada. Recuperado de http://digibug. ugr.es/bitstream/10481/2733/1/18505685.pdf.

Jiménez Mendoza, W., Soto Carrión, C., \& Urrutia Huamán, R. (2015). Relación entre modelo PIHEM y gestión educativa en instituciones educativas del nivel inicial en el distrito de Abancay Apurímac Perú, 2014. Revista De Investigaciones UNAD, 14(1), 81 95. doi:http://dx.doi.org/10.22490/25391887.1347

Jouini, K. (2005). Estrategias inferenciales en la comprensión lectora. Glossas didácticas, 9, 95-104. Recuperado de http://www.um.es/glosasdidacticas/GD13/GD13_10.pdf.

Krashen, S. \& Terrel, T. (1983). The natural approach. Language acquisition in the Classroom. Recuperado de http://www.osea-cite.org/class/SELT_materials/SELT_ Reading_Krashen_.pdf.

Lurán Rivero, A., Buenahora Tobar, M., \& Vargas Granados, C. (2015). Perspectiva del aprendizaje tradicional y del aprendizaje con comunicación bidireccional en actividades educativas. Revista De Investigaciones UNAD, 14(1), 253-262. doi:http://dx.doi. org/10.22490/25391887.1468

McNamara, D. S. (2004). SERT: Self-explanation reading training. Discourse Processes, 38 , 1-30. Doi:10.1207/s15326950dp3801_1Danielle S. McNamara.

Ministerio de Educación Nacional (febrero 8 de 1994). Ley 115. Recuperado de http:// www.mineducacion.gov.co/1621/article-85906.html

Ministerio de Educación Nacional (2006). Serie guía 22/ Estándares básicos de competencias en lenguas extranjeras: inglés. Bogotá: Ministerio de Educación Nacional. Recuperado de http://www.mineducacion.gov.co/cvn/1665/articles-115174_archivo_ pdf.pdf

Ministerio de Educación Nacional (2014). Programa Nacional de inglés 2015-2025. Bogotá: Ministerio de Educación Nacional. Colombia Very well!. Recuperado de http:// www.colombiaaprende.edu.co/html/micrositios/1752/articles-343287_recurso_1.pdf

Muñoz Restrepo, A. (2009). Metodología para la enseñanza de las lenguas extranjeras: hacia una perspectiva crítica. Revista Universidad EAFIT, 46(159), 71-85. Recuperado de http://publicaciones.eafit.edu.co/index.php/revista-universidad-eafit/article/ viewFile/1065/959.

Sánchez, E (2009). Mente, cerebro y educación. Revista de pedagogía de la Universidad de Salamanca, 15, 25-46. Recuperado de http://campus.usal.es/ revistas_trabajo/ index.php/0214-3402/article/view/6947/10425.

Shang, H. (2006). Content-based Instruction in the EFL Literature Curriculum: TESL Journal. Vol. XII. No. 11 Recuperado de http://iteslj.org/Techniques/Shang-CBI.html

Silva Carreño, W., \& Beltrán Martin, J. (2015). El rol de género como fundamento humanista de la formación para la ciudadanía. Revista De Investigaciones UNAD, 14(1), 7 - 17. doi:http://dx.doi.org/10.22490/25391887.1343 
Solé, I. (1998). Estrategias de lectura. Recuperado de https://media.utp.edu.co/ referencias-bibliograficas/uploads/referencias/libro/1142-estrategias-de-lecturapdfNOaU6-libro.pdf

Van Dijk, T. A. \& Kintsch, W. (1983). Strategies of discourse comprehension. New York: Academic Press. Recuperado en http://www.discourses.org/OldBooks/Teun\%20A\%20 van\%20Dijk\%20\%26\%20Walter\%20Kintsch\%20-\%20Strategies\%20of\%20Discourse\%20 Comprehension.pdf

Yin, R. K. (1981). Case Study Research. London: Sage Publications. Recuperado de http://www.madeira-edu.pt/LinkClick.aspx?fileticket=Fgm4GJWVTRs\%3D\&tabid=3004 